DOI: 10.34031/article_5d4d6a32d95185.53258375

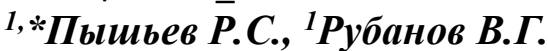

${ }^{I}$ Белгородский государственный технологический университет им. В.Г. Шухова

Россия, 308012, г. Белгород, ул. Костюкова, д. 46

*E-mail:dobe.87@mail.ru

\section{НЕЧЕТКАЯ ЭКСТРЕМАЛЬНАЯ СИСТЕМА АВТОМАТИЧЕСКОЙ НАСТРОЙКИ РЕЗОНАНСНОГО КОНТУРА}

Аннотация. В робототехнике радиопередача может использоваться в системах связи, которые являются неотъемлемой частью роботов. С помощьюю систем связи осуществляется обмен информацией между роботом и человеком, формирование задач, контроль функиионирования остальньх систем, диагностика неполадок и т.п. При формировании управляющчих сигналов возникает необходимость воспрепятствовать прохождению сигнала определенной частоты, пропуская другие сигналь, например, убрать помеху на определенной частоте или наоборот, заземлить все сигналь, кроме нужного, данной частоты. Статическая характеристика контура представляет собой параболическую зависимость с наличием экстремума на резонансной частоте. При этом, изменяя параметры колебательного контура или частоту подаваемого на контур напряжения, можно наблюдать горизонтальный дрейф амплитудно-частотной характеристики контура. Построение систем экстремального регулирования (СЭР) способствует адаптации к изменениям и дрейфу статических характеристик. Основным недостатком СЭР является наличие частых переключений исполнительных механизмов, сопутствуюших поисковому режиму, и так называемых потерь на «поиск» и «рыскание», что в итоге приводит к снижению эффективности автоматической настройки и надежности устройств для ее осуществления. Экстремальные комбинированные системы используют свойства статической характеристики объекта регулирования, и их разработка является одним из путей снижения указанньх негативных качеств.

При создании систем автоматического управления для сложных технологических объектов в условиях большой неопределенности и неполноты знаний об объекте, нечеткости описаний, как показывает опыт, применение только классических формальных методов теории управления не эффективно. Всё больше наблюдается повышенный интерес к невероятностным моделям неопределенности и нечеткости, в частности к анализу и синтезу нечетких систем управления технологическими прочессами и регуляторов.

Ключевые слова: колебательный контур, экстремальная система, нечеткая система.

Введение. Элементы, составляющие колебательный контур, способны запасать магнитную и электрическую энергию. В электрической цепи, состоящей из таких элементов, возбуждаются электрические колебания. В системах, у которых амплитуда отклика цепи может резко измениться, при достижении некоторых значений частоты внешнего воздействия, определяемых параметрами цепи, нашли широкое применение колебательные контуры. Подобные системы называются частотно-избирательными и зачастую применяются в радиоэлектронике [1]. Простым примером колебательного контура является последовательное или параллельное соединение двух реактивных сопротивлений противоположного знака: индуктивного XL и емкостного XC.

Частотные характеристики параллельного колебательного контура представлены на рисунке 1 .

Как видно, АЧХ представляет собой параболическую зависимость с наличием экстремума на резонансной частоте. При этом, изменяя параметры колебательного контура или частоту пода- ваемого на контур напряжения, можно наблюдать горизонтальный дрейф амплитудно-частотной характеристики контура.

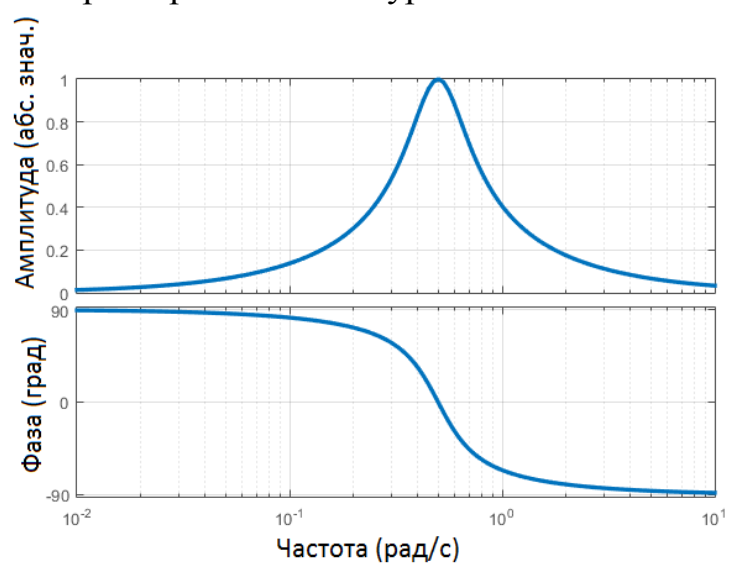

Рис. 1. Амплитудно- и фазо- частотные характеристики параллельного колебательного контура

Построение систем экстремального регулирования (СЭР) способствует адаптации к изменениям и дрейфу статических характеристик. Ос- 
новным недостатком СЭР является наличие частых переключений исполнительных механизмов, сопутствующих поисковому режиму, и так называемых потерь на «поиск» и «рыскание», что в итоге приводит к снижению эффективности автоматической настройки и надежности устройств для ее осуществления. Экстремальные комбинированные системы используют свойства статической характеристики объекта регулирования, и их разработка является одним из путей снижения указанных негативных качеств [2].

При создании систем автоматического управления для сложных технологических объектов в условиях большой неопределенности и неполноты знаний об объекте, нечеткости описаний, как показывает опыт, применение только классических формальных методов теории управления не эффективно. Всё больше наблюдается повышенный интерес к невероятностным моделям неопределенности и нечеткости, в частности к анализу и синтезу нечетких систем управления технологическими процессами и регуляторов [3].

В отличие от традиционных формально-логических систем нечеткая логика, служащая основой для реализации методов нечеткого управления, описывает характер человеческого мышления и ход его рассуждений более естественно. Именно поэтому изучение и использование математических средств для представления нечеткой исходной информации позволяет строить модели, отражающие различные аспекты неопределенности наиболее адекватно [4].

Сроки и стоимость проектирования нечетких систем управления, как показывает практический опыт разработки таких систем, значительно ниже, чем при использовании традиционного математического аппарата, при этом организация нечеткого управления обеспечивает требуемые уровни качества [4].

Методология. Существует два основных метода построения систем автоматического регулирования [5]:

- построение систем экстремального регулирования (СЭР), требующих введения процедуры поиска;

- организация систем управления по отклонению, требующих детального изучения объекта управления и наличия достаточной информации на выходе объекта.

Реализация системы автоматического регулирования по отклонению часто связана с серьезными аппаратурными и измерительными трудностями. Создать систему экстремального управления часто оказывается проще, чем систему авто- матического регулирования, поскольку экстремальная система не требует особо тщательной организации сбора информации о состоянии объекта и соответствующей обработки этой информации. Совершенство системы автоматического регулирования по отклонению возможно за счет еe высокой организации, которая неизбежно имеет пониженную надежность и всегда таит опасность возникновения неустойчивости [5]. Оба метода имеют свои достоинства и недостатки. Выбор той или иной схемы управления нужно производить исходя из конкретных условий и требований.

Основная часть. Функциональная схема системы автоматического регулирования экстремального класса представлена на рисунке 2.

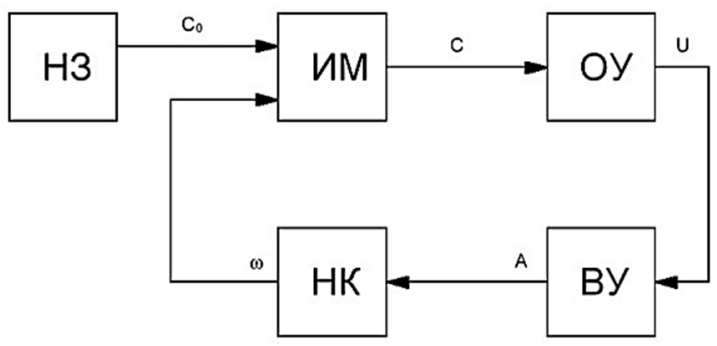

Рис. 2. Структурная схема системы автоматической настройки колебательного контура в резонанс: Н3 - начальное значение емкости конденсатора $\mathrm{C}_{0} ;$ ИМ - исполнительный механизм; ОУ - объект управления; ВУ - вычислительное устройство; НК - нечеткий контроллер

Резонансный контур настраивается с помощью переменного конденсатора С. Для настройки контура необходимо установить такое значение емкости $\mathrm{C}$, чтобы резонансная частота контура и частота поступающего на контур сигнала совпали. Признаком такой настройки является максимум напряжения на резонансном контуре. Таким образом, задача настройки сводится к определению значения $\mathrm{C}$, соответствующего максимуму напряжения на резонансном контуре (см. рисунок 2).

На рис. 3 представлена модель автоматического регулирования резонанса в колебательном контуре. Входное напряжение подаётся с помощью блока SineWave, в котором задается амплитуда и частота сигнала. Сигнал на выходе блока Integrator представляет собой выходное напряжение с контура. С помощью блока MinMax определяется амплитуда выходного напряжения. Блок Integrator2 используется в качестве конденсатора переменной емкости. Скорость вращения ротора конденсатора задается нечетким контроллером, имеющим 3 входа и один выход. 


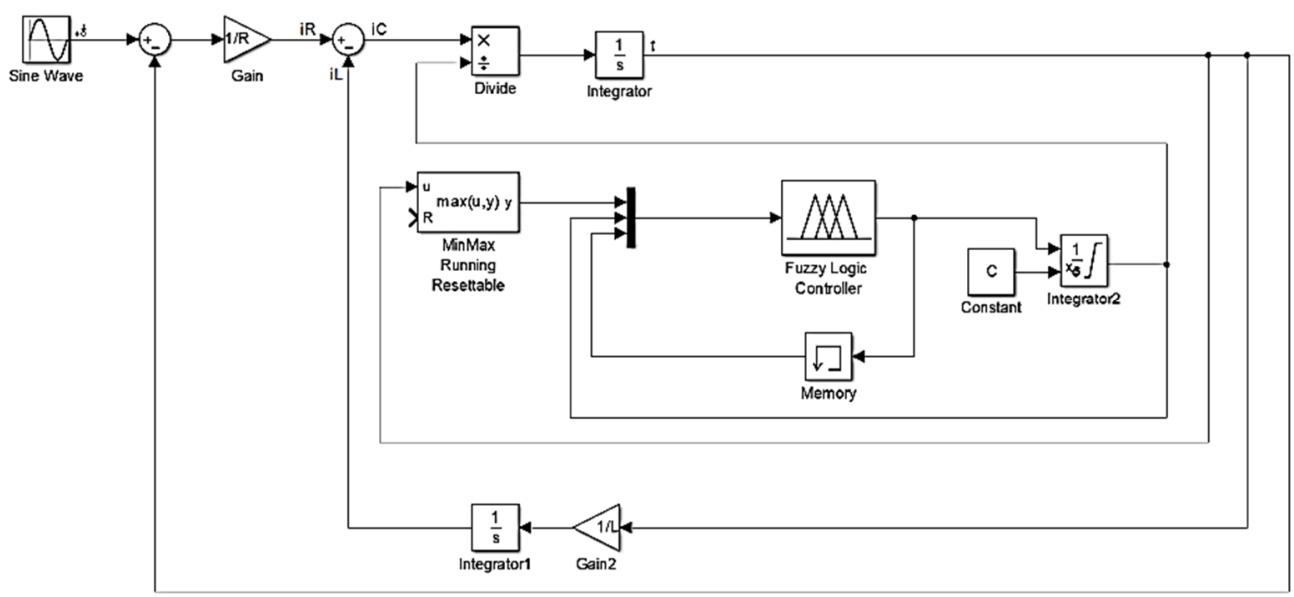

Рис. 3. Модель системы автоматической настройки колебательного контура в резонанс в среде Matlab/Simulink

Первый вход «Amplitude» - амплитуда напряжения на колебательном контуре. Имеет следующие термы:

$\mathrm{SA}$ - малая амплитуда, диапазон принадлежности $[0 . .0,4 \mathrm{~A}] \mathrm{B}$;

МА - средняя амплитуда, диапазон принадлежности $[0,4 \mathrm{~A} . .0,8 \mathrm{~A}] \mathrm{B}$;

ВА - большая амплитуда, диапазон принадлежности [0,7А .. А] В;

где А - амплитуда входного сигнала.

Второй вход «Сарасіtу» - значение емкости переменного конденсатора. Имеет следующие термы:

$\mathrm{SC}$ - малое значение емкости, диапазон принадлежности $\left[10^{-12} . .10^{-11}\right] \Phi$;

МC - среднее значение емкости, диапазон принадлежности $\left[5 \cdot 10^{-12} . .5 \cdot 10^{-7}\right] \Phi$;

$\mathrm{BC}$ - большое значение емкости, диапазон принадлежности $\left[10^{-7} . .10^{-6}\right] \Phi$.

Третий вход «PreviousSpeed» - предыдущее значение скорости регулирования. Имеет следующие термы:
NS -отрицательная скорость, диапазон принадлежности [-1 .. 0.01] Ф/c;

PS -положительная скорость, диапазон принадлежности [-0,01 .. 1] Ф/с.

Выход нечеткого контроллера «ActuatorSpeed»- скорость изменения емкости, имеет следующие термы:

BNS - большая отрицательная скорость, диапазон принадлежности [-1 .. -0.4] Ф/c;

SNS - малая отрицательная скорость, диапазон принадлежности [-0,5 .. -0.1] Ф/с;

AZS -скорость близкая к нулю, диапазон принадлежности [-0,2 .. 0.2] Ф/c;

SPS - малая положительная скорость, диапазон принадлежности $[0,1 . .0 .5] \Phi / \mathrm{c}$;

BPS - большая положительная скорость, диапазон принадлежности $[0.4$.. 1] Ф/c;

Нечеткий регулятор работает по алгоритму Мамдани, который представлен на рисунке 4, в соответствии с базой продукционных правил, представленной на рисунке 5.

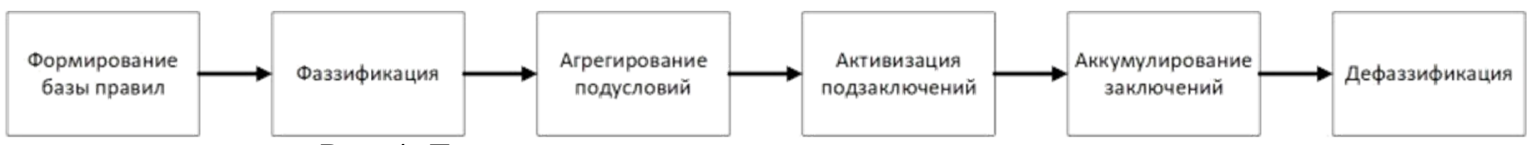

Рис. 4. Диаграмма деятельности процесса нечеткого вывода

PreviousSpeed - PS

\begin{tabular}{|c|c|c|c|}
\hline Capacity & SA & MA & BA \\
\hline SC & BPS & SPS & AZS \\
\hline MC & BPS & SPS & AZS \\
\hline BC & BNS & SNS & AZS \\
\hline
\end{tabular}

PreviousSpeed - NS

\begin{tabular}{|l|l|l|l|}
\hline \multicolumn{1}{|c|}{$\begin{array}{l}\text { Amplitude } \\
\text { Capacity }\end{array}$} & SA & MA & BA \\
\hline SC & BPS & SPS & AZS \\
\hline MC & BNS & SNS & AZS \\
\hline BC & BNS & SNS & AZS \\
\hline
\end{tabular}

Рис. 5. База продукционных правил нечеткого контроллера 

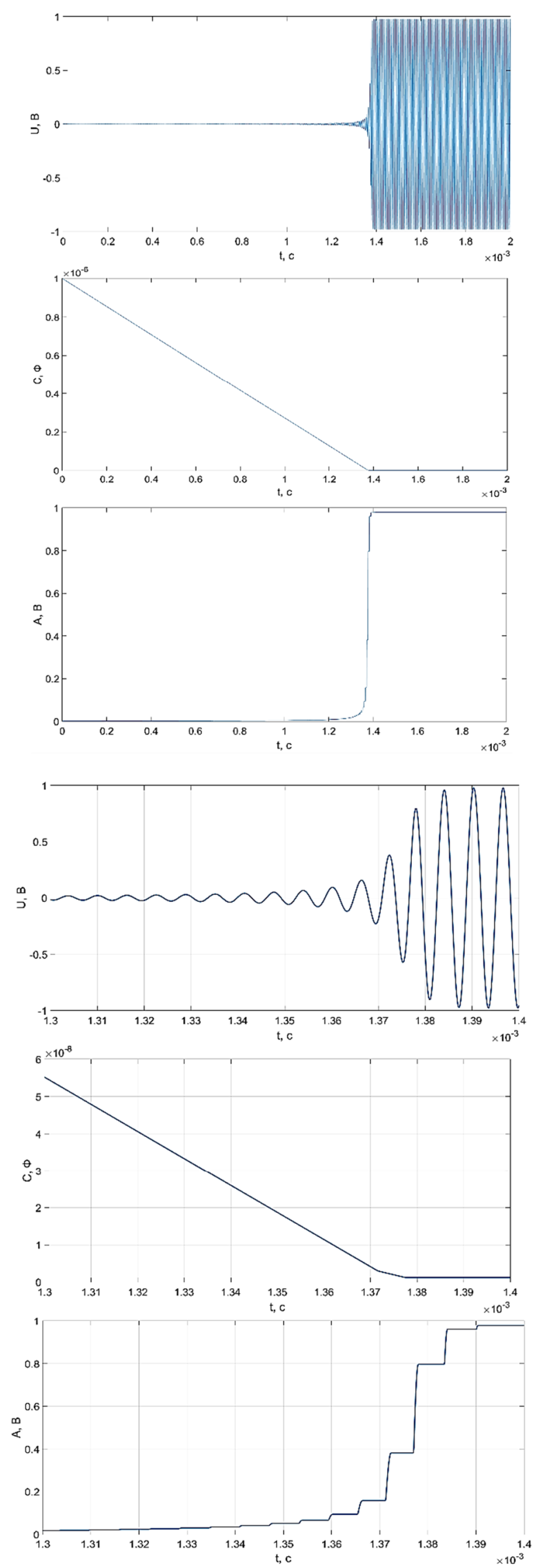

Рис. 6. Результат моделирования (полный график процесса и масштаб в момент резонанса). Верхний график - напряжение колебательного контура, средний - емкость конденсатора, нижний - амплитуда напряжения колебательного контура.
В качестве примера работы системы были заданы следующие параметры:

$\mathrm{R}$ - активное сопротивление - 1 кОм;

$\mathrm{L}$ - индуктивность катушки - 1 мГн;

C - начальное значение емкости конденсатора - 1 мкФ;

Амплитуда входного сигнала - 1 В;

Частота входного сигнала - 159 кГц.

Результат работы системы автоматической настройки колебательного контура в резонанс представлен на рисунке 6.

Выводы. Выполнен анализ систем автоматического регулирования, позволяющих решить задачу автоматической настройки колебательного контура в резонанс. Разработана функциональная схема нечеткой системы экстремального управления подстройкой колебательного контура. Получена математическая модель в виде передаточной функции объекта управления (колебательного контура) и системы автоматического регулирования в целом и построена структурная схема системы. Проведено экспериментальное исследование компьютерной модели нечеткой системы автоматической настройки колебательного контура в резонанс в среде Matlab/Simulink.

\section{БИБЛИОГРАФИЧЕСКИЙ СПИСОК}

1. Скрипников Ю.Ф. Колебательный контур. М., «Энергия», 1970. 128 с.

2. Бушуев Д.А. Система автоматического демпфирования колебаний помольно-смесительного агрегата на основе экстремального управления: автореф. дис. БГТУ им. В.Г. Шухова. Белгород, 2016. 183 с.

3. Кудинов Ю.И., Дорохов И. Н., Пащенко Ф.Ф. Нечеткие регуляторы и системы управления // Проблемы управления. 2004. №3. С. 2-14.

4. Леоненков А.В. Нечеткое моделирование в среде Matlab и fuzzyTЕСН. СПб.: БХВ-Петербург, 2005. 786 с.

5. Растригин Л.А. Системы экстремального регулирования. М.: Наука, 1974. 632 с.

6. Олейников В.А. Зотов Н.С., Пришвин А.М.. Основы оптимального и экстремального управления. М.: Высшая школа, 1969. 296 с.

7. Казакевич В.В., Родов А.Б. Системы автоматической оптимизации. М.: Энергия, 1977. 288 c.

8. Кунцевич В.М., Лычак М.М. Синтез оптимальных и адаптивных систем управления. Игровой подход.Киев: Наукова думка, 1985. 247 с.

9. А.с. 210918 СССР, МПК G 05 В 13/02. Экстремальный регулятор с запоминанием экстремума / Троп А.Е., Гафиятуллин Р.Х. 1129620/2624 ; заявл. 30.01.67; опубл. 8.01.68, Бюл. 7. 2 с. 
10. Солодовников В.В., Шрамко Л.С. Расчет и проектирование аналитических самонастраивающихся систем с эталонной моделью. М.: Машиностроение, $1972.270 \mathrm{c}$.

11. А. c. 817728 СССР, МПК G 05 В 13/02. Устройство для определения частной производной. / Востриков А.С., Хачатурова С.М., Гаврилов Е.Б., Гаврилов А.Б. 3375348/18-24; заявл. 6.01.82; опубл. 7.03.83, Бюл. 6.4 с.

12. А. с. 568952 СССР, МКИ G 06 G 7/18. Устройство для многократного дифференцирования аналоговых сигналов / Востриков А.С., Гаврилов Е.Б. 2302443/24; заявл. 15.12.75; опубл. 15.08.77, Бюл. 30.3 с.

13. Анисимов И.В. Основы автоматического управления технологическими процессами нефтехимической и нефтеперерабатывающей промышленности. Ленинград: Химия, 1967. $408 \mathrm{c}$.

14. Юревич Е. Теория автоматического управления, 4-е изд. Учебное пособие. БХВ-Петербург, 2016. $560 \mathrm{c.}$

15. Бушуев Д.А. Моделирование подсистемы регулирования положения противовеса помольно-смесительного агрегата с автоматической балансировкой/ Д.А. Бушуев, М.Ю. Лазарчук, С.А. Стативко // Сб. трудов международного форму «Образование, наука, производство». Белгород. 20-22 октября 2015 г., Белгород: Изд-во БГТУ им. В.Г. Шухова, 2015. С. 2765-2769.

Информация об авторах

Пышьев Роман Сергеевич, магистрант кафедры технической кибернетики. E-mail: dobe.87@mail.ru. Белгородский государственный технологический университет им. В.Г. Шухова. Россия, 308012, Белгород, ул. Костюкова, д. 46.

Рубанов Василий Григорьевич, доктор технических наук, профессор кафедры технической кибернетики. E-mail: vgrubanov@gmail.ru. Белгородский государственный технологический университет им. В.Г. Шухова. Россия, 308012, Белгород, ул. Костюкова, д. 46.

Поступила в мае 2019 г.

(C) Пышьев Р.С., Рубанов В.Г., 2019

1,*Pyshev R.S., ${ }^{1}$ Rubanov V.G.

${ }^{1}$ Belgorod State Technological University named after V.G. Shukhova

Russia, 308012, Belgorod, st. Kostyukova, 46

*E-mail:dobe.87@mail.ru

\title{
FUZZY EXTREME SYSTEM OF AUTOMATIC ADJUSTMENT OF A RESONANCE CIRCUIT
}

\begin{abstract}
In robotics, radio transmission can be used in communication systems that are an integral part of robots and serve to organize the exchange of information between the robot and humans or other robots in order to carry out a dialogue, formulate tasks for the robot, monitor the functioning of its systems, diagnose faults, routine checks, etc. The resonant circuit is used if it is necessary to prevent the passage of a signal of a certain frequency, passing other signals, for example, to remove the interference at a certain frequency or vice versa, to ground all signals except the desired frequency. The static characteristic of the circuit is a parabolic dependence with the presence of an extremum at the resonant frequency. In this case, by changing the parameters of the oscillating circuit or the frequency of the voltage applied to the circuit, one can observe the horizontal drift of the amplitude-frequency characteristic of the circuit. Adaptation to changes in static characteristics and their drift contributes to the construction of systems of extreme regulation. The main disadvantage of such systems is the presence of frequent actuators accompanying the search mode and the so-called "search" and "yaw" losses, which ultimately leads to a decrease in the efficiency of automatic tuning and reliability of devices for its implementation. One of the ways to reduce these negative properties is the development of extreme combined systems that use the properties of the static characteristics of the object of regulation. There is an increasing interest in improbable models of uncertainty and fuzziness, in particular, in the analysis and synthesis of fuzzy process control systems and regulators. The time and cost of designing fuzzy control systems, as shown by practical experience in developing such systems, is significantly lower than when using the traditional mathematical apparatus, and the organization of fuzzy control provides the required quality levels.
\end{abstract}

Keywords: resonant circuit, extreme system, fuzzy system. 


\section{REFERENCES}

1. Skripnikov U.F. Reconant circuit [Kolebatel'nyj kontur]. M., Energiya, 1970. 128 p. (rus)

2. Bushuev D.A. Automatic vibration damping system for the grinding and mixing unit based on extreme control [Sistema avtomaticheskogo dempfirovaniya kolebanij pomol'no-smesitel'nogo agregata na osnove ekstremal'nogo upravleniya]: avtoref. dis. BGTU named after V.G. Shuhov. Belgorod, 2016. 183 p. (rus)

3. Kudinov U.I., Dorohov I.N., Pashchenko F.F. Fuzzy controllers and control systems [Nechetkie regulyatory i sistemy upravleniya]. Problemy upravleniya. 2004. No. 3. Pp. 2-14. (rus)

4. Leonenkov A.V. Fuzzy simulation in Matlab and fuzzyTECH [Nechetkoe modelirovanie $\mathrm{v}$ srede Matlab i fuzzyTECH]. SPb.: BHV-Peterburg, 2005. 786 p. (rus)

5. Rastrigin L.A. Extreme regulation systems [Sistemy ekstremal'nogo regulirovaniya]. M.: Nauka, 1974. 632 p. (rus)

6. Olejnikov V.A., Zotov N.S. Prishvin A.M. Basics of optimal and extreme control [Osnovy optimal'nogo i ekstremal'nogo upravleniya].. M.: Vysshaya shkola, 1969. 296 p. (rus)

7. Kazakevich V.V., Rodov A.B. Auto Optimization Systems [Sistemy avtomaticheskoj optimizacii]. M.: Energiya, 1977. 288 p. (rus)

8. Kuncevich V.M., Lychak M.M. Synthesis of optimal and adaptive control systems. Game method [Sintez optimal'nyh i adaptivnyh sistem upravleniya. Igrovoj podhod]. Kiev: Naukova dumka, 1985. 247 p. (rus)

9. A.s. 210918 SSSR, MPK G 05 V 13/02. Extreme Memory Controller [Ekstremal'nyj regulyator s zapominaniem ekstremuma]. Trop A.E., Gafiyatullin R.H. 1129620/26-24, 1968.
10. Solodovnikov V.V., SHramko L.S. Calculation and design of analytical self-adjusting systems with a reference model [Raschet i proektirovanie analiticheskih samonastraivayushchihsya sistem s etalonnoj model'yu]. M.: Mashinostroenie, 1972. 270 p. (rus)

11. A. s. 817728 SSSR, MPK G 05 V 13/02. Device for the determination of private derivative [Ustrojstvo dlya opredeleniya chastnoj proizvodnoj]. Vostrikov A.S., Hachaturova S.M., Gavrilov E.B., Gavrilov A.B. 3375348/18-24, 1983.

12. A. s. 568952 SSSR, MKI G 06 G 7/18. Device for multiple differentiation of analog signals [Ustrojstvo dlya mnogokratnogo differencirovaniya analogovyh signalov]. Vostrikov A.S., Gavrilov E.B. 2302443/24, 1977.

13. Anisimov I.V. Basics of automatic control of technological processes in the petrochemical and refining industries [Osnovy avtomaticheskogo upravleniya tekhnologicheskimi processami neftekhimicheskoj i neftepererabatyvayushchej promyshlennosti]. Leningrad.: Himiya, 1967. 408 p. (rus)

14. Urevich E. Automatic control theory [Teoriya avtomaticheskogo upravleniya], 4-e izd. Uchebnoe posobie. BHV-Peterburg, 2016. 560 p. (rus)

15. Bushuev D.A., Lazarchuk M.Yu., Stativko S.A. Simulation of the subsystem for controlling the position of the counterweight of the grinding and mixing unit with automatic balancing [Modelirovanie podsistemy regulirovaniya polozheniya protivovesa pomol'no-smesitel'nogo agregata $\mathrm{s}$ avtomaticheskoj balansirovkoj]. Sb. trudov mezhdunarodnogo formu «Obrazovanie, nauka, proizvodstvo». Belgorod: Bulletin of BSTU named after V.G. Shukhov, 2015. Pp. 2765-2769.

Information about the authors

Pyshev, Roman S. Master student. E-mail: dobe.87@mail.ru.Belgorod State Technological University named after V.G. Shukhov. Russia, 308012, Belgorod, st. Kostyukova, 46.

Rubanov, Vasiliy G. DSc, Professor. E-mail: vgrubanov@gmail.ru. Belgorod State Technological University named after V.G. Shukhov.Russia, 308012, Belgorod, st. Kostyukova, 46.

Received in May 2019

\section{Для цитирования:}

Пышьев Р.С., Рубанов В.Г. Нечеткая экстремальная система автоматической настройки резонансного контура // Вестник БГТУ им. В.Г. Шухова. 2019. № 8. С. 115-120. DOI: $10.34031 /$ article_5d4d6a32d95185.53258375

\section{For citation:}

Pyshev R.S., Rubanov V.G. Fuzzy extreme system of automatic adjustment of a resonance circuit. Bulletin of BSTU named after V.G. Shukhov. 2019. No. 8. Pp. 115-120. DOI: $10.34031 /$ article_5d4d6a32d95185.53258375 